Meta

Journal des traducteurs

Translators' Journal

\title{
L'exportation du modèle terminologique québécois en Catalogne
}

\section{Jean Quirion et Judit Freixa}

Volume 58, numéro 3, décembre 2013

URI : https://id.erudit.org/iderudit/1025056ar

DOI : https://doi.org/10.7202/1025056ar

Aller au sommaire du numéro

Éditeur(s)

Les Presses de l’Université de Montréal

ISSN

0026-0452 (imprimé)

1492-1421 (numérique)

Découvrir la revue

Citer cet article

Quirion, J. \& Freixa, J. (2013). L'exportation du modèle terminologique québécois en Catalogne. Meta, 58(3), 643-661. https://doi.org/10.7202/1025056ar
Résumé de l'article

Dans les ensembles espagnol et canadien, la Catalogne et le Québec partagent plusieurs caractéristiques. Bénéficiant tous deux de pouvoirs politiques, économiques et linguistiques, ils ont mis à profit ces derniers pour les travaux d'aménagement linguistique et terminologique sur leurs territoires respectifs. En outre, les sociétés catalane et québécoise ont en commun que leur langue nationale est minoritaire au sein du pays, ce qui a engendré au fil du temps la mise en place de multiples mesures pour la préserver et la dynamiser. Le présent article retrace la forme qu'ont pris les nombreux échanges entre le Québec et la Catalogne depuis le milieu des années 1970, moment-clé pour l'affirmation linguistique des deux peuples. Les diverses facettes de l'apport du Québec à la Catalogne en ce qui touche l'aménagement linguistique sont plus particulièrement examinées. Ces échanges ont inspiré les Catalans dans la création de leur politique d'éducation et de normalisation terminologique, notamment ; citons entre autres l'Office québécois de la langue française qui trouve en Termcat un équivalent catalan depuis maintenant 25 ans. Également, en plus des idées, les autres formes des échanges québécois-catalans sont décrites. Un bilan des échanges Québec-Catalogne est ensuite dressé, notamment pour déterminer globalement l'impact qu'aura eu l'influence québécoise dans la discipline. La conclusion souligne que, depuis nombre d'années, la Catalogne rayonne fortement dans le domaine terminologique : elle est devenue elle-même un important lieu de diffusion vers d'autres communautés et constitue à son tour une source d'inspiration pour le Québec.
Ce document est protégé par la loi sur le droit d'auteur. L'utilisation des services d'Érudit (y compris la reproduction) est assujettie à sa politique d'utilisation que vous pouvez consulter en ligne.

https://apropos.erudit.org/fr/usagers/politique-dutilisation/ 


\title{
TERMINOLOGIE
}

\section{L'exportation du modèle terminologique québécois en Catalogne:}

\author{
JEAN QUIRION \\ École de traduction et d'interprétation, Université d'Ottawa, Canada \\ JQuirion@uottawa.ca
}

JUDIT FREIXA

Institut Universitari de Lingüística Aplicada, Universitat Pompeu Fabra, Barcelone, Espagne judit.freixa@upf.edu

\section{RÉSUMÉ}

Dans les ensembles espagnol et canadien, la Catalogne et le Québec partagent plusieurs caractéristiques. Bénéficiant tous deux de pouvoirs politiques, économiques et linguistiques, ils ont mis à profit ces derniers pour les travaux d'aménagement linguistique et terminologique sur leurs territoires respectifs. En outre, les sociétés catalane et québécoise ont en commun que leur langue nationale est minoritaire au sein du pays, ce qui a engendré au fil du temps la mise en place de multiples mesures pour la préserver et la dynamiser. Le présent article retrace la forme qu'ont pris les nombreux échanges entre le Québec et la Catalogne depuis le milieu des années 1970, moment-clé pour l'affirmation linguistique des deux peuples. Les diverses facettes de l'apport du Québec à la Catalogne en ce qui touche l'aménagement linguistique sont plus particulièrement examinées. Ces échanges ont inspiré les Catalans dans la création de leur politique d'éducation et de normalisation terminologique, notamment; citons entre autres l'Office québécois de la langue française qui trouve en Termcat un équivalent catalan depuis maintenant 25 ans. Également, en plus des idées, les autres formes des échanges québécois-catalans sont décrites. Un bilan des échanges Québec-Catalogne est ensuite dressé, notamment pour déterminer globalement l'impact qu'aura eu l'influence québécoise dans la discipline. La conclusion souligne que, depuis nombre d'années, la Catalogne rayonne fortement dans le domaine terminologique: elle est devenue ellemême un important lieu de diffusion vers d'autres communautés et constitue à son tour une source d'inspiration pour le Québec.

\section{ABSTRACT}

Within their respective countries, Catalonia and Quebec share many characteristics. Both have exercised their political, economic and linguistic powers to advance language planning and terminology planning in their region. Moreover, Catalan and Quebecois societies both have a language that is in the minority relative to the country to which they belong - a fact that, over time, has led to the implementation of multiple means of preserving and revitalizing these languages. This article outlines the numerous exchanges between Quebec and Catalonia since the mid-1970s, a key period in the linguistic assertion of both nations. Various aspects of the contribution Quebec has made to Catalonia are discussed with respect to language planning in particular. These exchanges inspired Catalans in the creation of their policy on education and terminology standardization: for example, the Office québécois de la langue française (i.e. the Quebec board of the French language) has, for the last 25 years, found its Catalan counterpart in Termcat. Furthermore, beyond 
the underlying ideas, the article describes the concrete manifestations of CatalanQuebecois sharing. The exchanges between Quebec and Catalonia are then summarized, notably so that the overall impact that Quebec has had within the discipline can be determined. The conclusion emphasizes that, for many years, Catalonia has exerted a strong influence on the field of terminology: it has itself become an important hub for disseminating terminological information to other communities and thus now constitutes a source of inspiration for Quebec.

\section{MOTS-CLÉS/KEYWORDS}

terminologie, langue d'usage, Québec, Catalogne, aménagement linguistique terminology, language of use, Quebec, Catalonia, language planning

\section{Le contexte}

Comme nous le verrons tout au long du présent article, le Québec et la Catalogne partagent de nombreuses similitudes, tant culturelles que linguistiques. Les deux régions ont connu une transformation rapide de leur situation politique et linguistique. Le Québec aura traversé, au tournant des années 1960, ce qu’on appelle la Révolution tranquille. En Catalogne, la fin du régime dictatorial de Franco aura permis aux catalanophones de prendre en main leur destinée linguistique.

\subsection{La situation québécoise}

Les années 1960 et 1970 ont vu naître un puissant sentiment nationaliste chez les francophones québécois; cette idée a d'ailleurs laissé des traces linguistiques: à cette époque, on a cessé d'utiliser province de Québec et Canadiens français pour parler respectivement du Québec et de ses habitants, les Québécois. À partir de cette période, le Québec est passé d'une dominance par la minorité anglophone riche, puissante et organisée à une appropriation progressive par les locuteurs francophones des leviers économiques, politiques et culturels.

À compter du début des années 1970, la législation vient sceller cette volonté d'un statut renforcé pour le français au Québec. La première loi linguistique d'importance, la loi 22, propulsant le français comme langue officielle du Québec, a été adoptée en 1974. Deux ans plus tard, en novembre 1976, le gouvernement nationaliste du Parti Québécois était élu. Dès l'année suivante, il promulgue la Charte de la langue française, communément appelée Loi 101. Cette dernière jetait les bases de la politique qui allait changer le visage linguistique du Québec. En effet, la Charte de la langue française stipulait que le français devait se généraliser comme la langue utilisée dans toutes les sphères de la vie, que ce soit le travail, les affaires, l'éducation, les loisirs, etc. Comme le signale Leclerc:

La stratégie linguistique de la Charte de la langue française reposait sur trois principes généraux visant à corriger les problèmes qui traînaient en longueur depuis plusieurs décennies:

- endiguer le processus d'assimilation et de minorisation des francophones;

- assurer la prédominance socio-économique de la majorité francophone;

- réaliser l'affirmation du fait français. 


\subsection{La situation catalane}

À l'heure actuelle, la région catalane se répartit en trois États européens différents: l'Espagne, la France et Andorre. C'est dans le premier que sont concentrés près de $95 \%$ du territoire et du nombre de locuteurs; dans les circonstances, le présent article se référera exclusivement à l'Espagne.

Les Catalans s'inscrivent déjà comme peuple quelques siècles avant la création de l'Espagne. Jusqu'en 1516, avec le mariage d'Isabelle de Castille et de Ferdinand du royaume catalano-aragonais, la Catalogne existe comme unité ou comme partie de la couronne catalano-aragonaise. Entre 1516 et 1714, la Catalogne vit deux siècles pendant lesquels elle partageait ses rois avec la Castille, mais la castillanisation se produit seulement au niveau aristocratique.

Pendant la Guerre de la Succession d'Espagne (1705-1714), la Catalogne appuie Charles d'Autriche, qui sera vaincu par Philippe d'Anjou, ce qui amènera une castillanisation progressive de la Catalogne. L’année 1716 marque la première exclusion du catalan dans l'Administration: le Decreto de Nueva Planta abolit la Generalitat (gouvernement de Catalogne) et les régimes juridique et fiscal propres à la Catalogne. Le catalan continue d'être l'idiome du peuple sans constituer la langue officielle, mais la substitution du catalan par le castillan dans les institutions administratives et scolaires signifie, dans les faits, le début d'une perte de reconnaissance de la spécificité catalane.

Dans la deuxième moitié du XIX $x^{\mathrm{e}}$ siècle nait le mouvement Renaixença et, avec lui, une idéologie nationaliste, qui défend la catalanité dans les domaines politique et culturel. La récupération s'avère lente et progressive jusqu'à ce que, au $\mathrm{Xx}^{\mathrm{e}}$ siècle, soient instaurées les dictatures de Primo de Rivera (1923), puis de Franco (1939). Entre ces deux totalitarismes, la Deuxième République espagnole (1931-1939) voit un bref regain catalan, qui prend fin abruptement avec la prise du pouvoir par Franco après la Guerre civile (1936-1939). Cette dictature, qui se prolongera jusqu'à sa mort en 1975, supprime les institutions républicaines et autonomes de la Catalogne, les partis catalanistes et prohibe l'usage officiel et public de l'idiome catalan. Cette politique anticatalane se traduit par la destruction de milliers de livres écrits en catalan, par l'exil forcé hors de Catalogne de nombreux professeurs catalans de l'enseignement primaire - qu'on remplaça par d'autres venus de Castille -, par l'épuration du corps professoral universitaire et par l'interdiction de l'académie de la langue, l'Institut d'Estudis Catalans (Bastardas Boada1987: 138).

Dans ce contexte, il convient de souligner que la Catalogne est une nation millénaire avec une langue propre et que ce n'est que depuis 100 ans, au début du $\mathrm{xx}^{\mathrm{e}}$ siècle, que la castillanisation de la population en général a commencé.

\subsection{Québec et Catalogne: des contextes différents}

Comme il a été dit précédemment, le Québec et la Catalogne offrent de nombreuses ressemblances, mais les différences entre les deux contextes sont également appréciables et expliquent que leurs routes ne soient pas toujours parfaitement parallèles. Nous nous baserons sur les propos d'auteurs comme Cabré (2001), Corbeil (1987; 1990) Reniu i Tresserras (2002) et Streicher-Arseneault (2010 et 2011) pour souligner les principaux contrastes que présentent les deux contextes à divers niveaux. 
D’abord, en ce qui a trait aux similitudes, il faut signaler le fait que le Québec et la Catalogne vivent tous deux une situation minoritaire: ce sont des nations à l'intérieur d'un État, bénéficiant d'un certain degré d'autonomie politique. Elles partagent également la caractéristique que leurs langues sont romanes (le français et le catalan respectivement) et en situation de contact avec une autre (l'anglais et le castillan respectivement). Ces dernières, tant au Canada qu'en Espagne, jouissent de la position d'idiome dominant. Enfin, tant le français que le catalan sont bien implantés sur leur territoire respectif et sont soumis à des politiques linguistiques de façon à les promouvoir et à les renforcer comme signes identitaires.

Les différences, de leur côté, se révèlent plutôt diversifiées. D’un point de vue historique, la Catalogne est une nation millénaire, marquée par l'établissement, puis l'affirmation de son identité. Pour sa part, le Québec résulte d'entreprises de colonisation menées au nord de l'Amérique il y a quatre siècles. La Catalogne s'inscrit dans le contexte européen, historiquement multiculturel et multilingue, tandis que le Québec se situe dans un pays où cohabitent principalement deux nations, dans un milieu anglophone certes, mais à la différence importante que sa langue trouve un large rayonnement extérieur. Pour cette raison, le statut des deux idiomes constitue une des distinctions majeures entre les deux peuples. Jamais le catalan n'aura obtenu de reconnaissance internationale complète, tandis que le français est officiel dans une cinquantaine d'États de par le monde.

Si l'on considère maintenant la perspective historique de la langue, le français parlé sur le territoire québécois n'a pas été autant ostracisé que le catalan a pu l'être. De plus, législativement, il importe de souligner que le cadre constitutionnel offre au Québec un pouvoir décisionnel plus étendu que celui de la Catalogne.

Le français cohabite au Québec avec l'anglais, langue internationale qui a préséance dans les mondes scientifique, technique et professionnel. En Catalogne, le catalan est en concurrence avec le castillan, mais également avec l'anglais, justement à cause du caractère hégémonique de ce dernier dans les sphères spécialisées. Enfin, sociolinguistiquement, l'anglais jouit d'un certain prestige au Québec, ce qui n'est pas exactement le cas du castillan en terre catalane.

\section{Les moyens déployés au Québec, sources d'inspiration en Catalogne}

Au cours du Xx $x^{\mathrm{e}}$ siècle, les nouvelles donnes linguistiques au Québec et en Catalogne ont engendré de grands besoins et nourri de vastes ambitions. Or, tout était à bâtir dans les deux États pour atteindre les nouveaux objectifs fixés. En effet, on manquait cruellement d'institutions, de moyens et de méthodes pour concrétiser les volontés des législateurs concernant la langue. Les circonstances historiques ayant voulu que le Québec traverse le premier cette phase de transformations linguistiques, c'est à lui qu'est revenu l'honneur d'innover dans ce domaine.

\subsection{Organisations}

Après la Charte de la langue française, le Québec adoptera progressivement d'autres lois à incidences linguistiques portant sur la protection du consommateur et sur l'affichage. Déjà, l’Office de la langue française, créé en 1961, surveillait de près les questions liées à la langue et guidait le gouvernement sur celles-ci. Plus tard, des 
institutions viennent s'ajouter afin d'appuyer l'Office dans ses activités: ainsi, le Secrétariat à la politique linguistique verra le jour, tout comme le Conseil supérieur de la langue française et la Commission de toponymie. Le premier assumera la coordination et la promotion de la politique linguistique du Québec et le second donnera son avis au gouvernement sur les questions relatives au français au Québec. Quant au troisième, il veillera à la gestion des noms de lieux au Québec.

\subsection{Besoins}

Le législateur québécois cherchait particulièrement à permettre à tous les locuteurs francophones de gagner leur vie dans leur langue maternelle. Or, dans la quasi-totalité des cas, le marché du travail québécois, malgré la volonté exprimée par la Charte, n'était pas en mesure d'offrir un environnement satisfaisant, tout simplement parce que les termes français manquaient.

Les vastes entreprises de recension et de création de termes nécessitaient, on s'en rendit compte sans tarder, des méthodes qui n'existaient pas encore. On comprendra aisément qu'une évolution aussi rapide, d'une situation où le français prenait une place de second ordre à celle d'avant-scène qu'on lui réservait désormais, entrainait de multiples conséquences. Outre les modifications sur le lexique lui-même, singulièrement dans les diverses sphères d'activité spécialisées, les moyens et les méthodes de description de la langue générale comme de la terminologie ont fait l'objet d'une révision complète, voire d'une création ex nihilo. Une vaste réflexion s'imposait à cet égard.

\subsection{Réflexions, moyens et méthodes}

La contribution québécoise s'est manifestée de multiples façons, que ce soit par des réflexions novatrices ou par des moyens et méthodes actualisant les volontés politiques.

Les actions de l'Office de la langue française reposent sur deux processus fondamentaux: l'aménagement linguistique et l'aménagement terminologique. Notons au passage que ces termes ont été créés en français au Québec (Delisle 2008: 52-53); les penseurs québécois ont joué un rôle important dans le développement de la discipline. Parallèlement, dans les années 1970, l’Office a programmé (et coorganisé, notamment avec la Société des traducteurs du Québec) une série de colloques portant sur la terminologie et ses méthodes. Ces dernières ont été profondément influencées par les réflexions alors menées, tant au Québec qu'en Catalogne et ailleurs dans le monde. On oublie parfois jusqu'à quel point la terminographie et la terminologie font figure de jeunes disciplines; pourtant, les questions abordées dans ces colloques - tenus il y a moins de 40 ans - touchent des éléments fondamentaux du domaine. Ceux-ci couvrent la nature d'une définition, le contenu d'une fiche décrivant une notion, la néologie, la diffusion et l'implantation des unités lexicales, l'enseignement de la matière, etc. Les recherches québécoises ont également porté sur la recherche sur la néologie et sur les critères favorisant l'implantabilité des innovations lexicales. On aura compris que l'ensemble de ces travaux allait trouver une utilité immédiate dans les années suivant l'adoption de la Charte de la langue française.

Parallèlement à ces réflexions et au gigantesque effort fourni sur le corpus linguistique, l'État québécois coordonnait en parallèle des actions pour valoriser le 
statut du français sur son territoire. Les analyses ont mené à la création de la Commission de terminologie de l'Office de la langue française (aujourd'hui le Comité d'officialisation linguistique), qui a le pouvoir de recommander des termes et même, dans certaines circonstances, d'en rendre l'usage obligatoire. S'y greffent des commissions de terminologie ministérielles, de comités de terminologie interentreprises et de comités de francisation, afin de favoriser la coordination entre les spécialistes, les milieux de travail et les terminologues. Ces structures étaient appuyées par du matériel promotionnel linguistique et de multiples autres mécanismes, afin d'actualiser cette volonté exprimée de renforcer et de généraliser l'usage de la langue française au Québec.

L'apport du Québec se traduit enfin par la création et la consignation de nombreuses méthodes terminographiques. L'Office, particulièrement, a rédigé de nombreux guides de travail en terminologie, donc ceux portant sur les méthodes de travail thématique et ponctuel, jetant les bases de la terminographie telle qu'elle est encore largement pratiquée aujourd'hui. L'Office québécois de la langue française entreprit d'imposantes recherches lexicales, créant et enrichissant au passage un vaste répertoire informatisé (la Banque de terminologie du Québec, aujourd'hui appelée le Grand dictionnaire terminologique). D’ailleurs, à la même époque, TERMIUM, qui est devenue la banque de données terminologique et linguistique du gouvernement canadien, voyait le jour à l'Université de Montréal.

Enfin, largement grâce à l'expérience acquise et aux réflexions approfondies dans les sphères gouvernementales, l'enseignement de la terminologie commence à prendre forme au Québec. Les tout premiers cours de terminologie sont intégrés aux programmes universitaires de traduction dès le début des années 1970.

\subsection{Amorce de coopération Québec-Catalogne: les années 1980 vues du Québec}

Les premiers indices d'une coopération Québec-Catalogne sont décelés très tôt dans les processus enclenchés de part et d'autre de l'Atlantique. Un décret du Gouvernement du Québec de $1983^{1}$ mentionne que les premières rencontres entre les représentants catalans et québécois remontent à 1981 et que, deux ans plus tard, les relations québéco-catalanes se sont accélérées et consolidées:

Déjà dans les années 1980, le Québec et la Catalogne partageaient leur expertise en matière de politique linguistique [...]. En plus d'amorcer la relation entre le Québec et la Catalogne, ces échanges ont contribué au développement de modèles de référence pour la réalisation, entre autres de la politique linguistique catalane [...]. En mars 1983, le Québec et la Catalogne marquaient officiellement les débuts de la synergie institutionnelle entre les deux gouvernements par le biais d'échanges de lettres. Ces dernières précisaient les bases de cette coopération qui allait toucher «tous les domaines de leur compétence», soit la langue, la culture et l'éducation. (Ministère des Relations internationales du Québec 2011a) ${ }^{2}$

Cette collaboration aura eu des retombées concrètes. «L'entente entre la Direcció General de Política Lingüística de la Generalitat de Catalunya et l'Office de la langue française du Québec a été permanente et importante. Nous avons partagé de nombreuses stratégies [...]» affirme Miquel Reniu i Tresserras, ex-directeur général de la politique linguistique catalane (Reniu i Tresserras 2002: 186). 
Claire Martin, de l'Office de la langue française, note:

En 1984, nous avons reçu [...] une mission officielle de la Direction générale de la politique linguistique [DGPL] dont faisait partie Mme Aina Moll i Marquès, directrice générale de la DGPL. [...] Par la suite, on a pu assister au développement, en Catalogne, de structures officielles de recherche en terminologie (création, entre autres de Termcat: centre de terminologie de la Catalogne). L'accent a dès lors été mis sur une coopération à caractère terminologique entre le Québec et la Catalogne. (Martin, 1989; cité dans Bouchard et Gaumont 1989: 4)

Cette proximité s'inscrit dans la durée, comme le confirme le préambule de la reconduction d'une entente de coopération entre le gouvernement du Québec et le gouvernement autonome de la Catalogne en 1996: "Attendu que le Québec et la Catalogne ont développé depuis 1983 des relations étroites de coopération dans les domaines de la langue, de la culture et de l'éducation [...]». (Ministère de Relations internationales du Québec 2011b)³. Par la suite, «[1]'année 1987 a été marquée par la signature d'un protocole de coopération linguistique entre l'Office de la langue française et la Direction générale de la politique linguistique de la Generalitat de Catalunya [...]» (Martin 1989; cité dans Bouchard et Gaumont 1989: 4).

Enfin, l'année 1989 est témoin du premier voyage de délégués québécois en terre catalane. En effet, deux représentants de l'Office de la langue française examineront les stratégies d'animation utilisées pour assurer la catalanisation des entreprises et du milieu, de même que l'organisation des services linguistiques de la Generalitat de Catalunya. Ils échangeront également sur les méthodes de travail en terminologie. Les auteurs du rapport constatent que les missions des deux organismes linguistiques présentent nombre de parallèles: «les deux organismes cherchent, par divers moyens, à amener l'Administration publique de leur État respectif à généraliser l'utilisation de la langue qu'ils ont le mandat de promouvoir» (Bouchard et Gaumont 1989: 47). Plus loin, ils ajoutent:

Le Québec a déjà grandement inspiré la Catalogne, nous a-t-on dit. C'est tout à l'honneur du Québec, mais, à la suite de cette mission, nous en sommes venus à croire que le Québec pourrait aussi apprendre de l'expérience catalane, des stratégies de catalanisation adoptées dans les ministères. (Bouchard et Gaumont 1989: 47)

On constate donc que, dès les premières années des échanges, la Catalogne inspire à son tour le Québec.

\section{Les nouvelles responsabilités catalanes et l'inspiration québécoise}

À la mort du dictateur Franco en 1975, et après une période de transition, la démocratie a été rétablie en Espagne, tout comme la Generalitat de Catalunya, c'est-à-dire le gouvernement de la communauté autonome de Catalogne. Tout était en place pour que la Catalogne relève le défi de ses nouvelles responsabilités.

La revitalisation des langue et culture catalanes entraîne elle aussi l'adoption de multiples lois et la création de maintes institutions. À l'affût, les Catalans ont alors entrepris de balayer le paysage linguistique afin de déterminer si d'autres nations que la leur n'avaient pas vécu la même situation. Ils ont effectivement trouvé au Québec une source d'inspiration et de collaboration.

Le modèle québécois a exercé sur la Catalogne une influence notable, tant en ce qui a trait à la politique linguistique en général qu'à l'organisation de la terminologie 
en particulier, que ce soit pour la rédaction de lois ou la création d'organismes liés à la politique linguistique. Seront exposés plus loin, chronologiquement, les faits saillants de l'officialisation linguistique en Catalogne, avec un accent spécial sur l'analyse de l'apport du Québec.

\subsection{L'aspect législatif}

La Constitution espagnole a été approuvée en 1978; elle représente le point de départ de l'aménagement linguistique (voir Vila i Moreno et Freixa (1990) pour une perspective générale sur l'aménagement linguistique en Espagne). L'article 3 de cette Constitution établit un système d'idiomes coofficiels: le castillan sera la langue officielle de la totalité de l'État espagnol et le catalan le sera tout autant en Catalogne . $^{4}$ Rappelons ici que le modèle québécois dont la Catalogne s'inspirera repose sur des textes législatifs qui correspondent à une situation linguistique fort différente ${ }^{5}$.

La constitution espagnole reconnaît, dans son article 147, que les communautés espagnoles disposent d'un texte juridique essentiel : l'Estatut d'Autonomia. Celui de la Catalogne a été approuvé en $1979^{6}$, à la suite d'un référendum, et fut ratifié par les Cortes Generales Españolas (Congrès et Sénat). Dans ce texte se retrouve la coofficialité constitutionnelle, mais à laquelle s'ajoute pour le catalan la valeur de langue propre de la Catalogne et, avec elle, la nécessité de faire du catalan l'idiome d'usage normal de la Catalogne (selon l'Estatut catalan et non l'acte espagnol). De ces balises découleront les lois d'officialisation linguistique qui, comme l'ont affirmé plusieurs spécialistes, s’inspirent directement de l'expérience québécoise:

L'influence québécoise est décelable dans de nombreux aspects de la politique linguistique catalane: l'implantation progressive du système d'immersion linguistique dans l'enseignement, par exemple, ou l'implantation d'un service public de consultations sur la langue et l'établissement de bases de données sur la terminologie, la recherche sociolinguistique, la création de services linguistiques dans le secteur syndical et l'entreprise, les différentes stratégies de signalisation extérieure, ou encore tout ce qui fait référence aux politiques de mise en valeur de la langue propre comme principal objectif pour son usage social. (Reniu i Tresserras 2002: 186-187)

L'organe étatique chargé de diriger, planifier, coordonner et mettre en œuvre la politique linguistique de la Generalitat de Catalogne est la Direcció General de Política Lingüística (direction générale de la politique linguistique); créée en 1980, elle est devenue en 2004 le Secretaria de Política Lingüística (Secrétariat à la politique linguistique), pour finalement reprendre son nom original en 2011. En revanche, son mandat, lui, n’a pas changé: assurer la mise en œuvre et le succès de la législation sur la langue, dérivée de l'Estatut.

Le point marquant des efforts catalans pour la récupération des droits linguistiques a eu lieu en 1983, au moment de l'approbation de la Llei de Normalització Lingüística (loi d'aménagement linguistique ${ }^{7}$ ). L’inspiration québécoise sur la rédaction de cette loi est reconnue:

L'influence de la Charte de la langue française du Québec sur la Llei de Normalització Lingüística (Loi 7/1983) et sur la réglementation catalane en vigueur est visible [...]. On retrouve également ce type d'effet des mesures prises en faveur du français dans les secteurs socioéconomiques et de l'enseignement sur la législation catalane, de 1983 à nos jours. (Reniu i Tresserras 2002: 187) 
Cette loi stipule que le catalan, à titre de langue propre de la Catalogne, doit être l'idiome normal d'enseignement à tous les niveaux du système éducatif (article 14.1). À cette époque, le Tribunal Constitucional espagnol reconnaissait la validité de cet article, aujourd'hui remis en cause, comme nous le verrons plus loin.

Le Consorci per a la Normalització Lingüística a été créé en 1988 avec comme objectif de favoriser l'enseignement du catalan chez la population adulte, en plus d'offrir une évaluation linguistique aux particuliers comme aux entreprises et de soutenir la promotion du catalan dans l'usage.

L'influence québécoise se fait également sentir, en 1993, lors de l'approbation de l'Estatut del consumidor (statut du consommateur). Selon Delisle, «[1]a loi sur la normalisation linguistique (1983) s'inspire directement de la Charte de la langue française du Québec, tout comme le statut du consommateur (Estatut del Consumidor, 1993) et la loi sur la politique linguistique (Llei de Política Lingüística, 1998).» (Delisle 2008: 189).

Justement, en 1998, est adoptée la Llei de Política Lingüística (loi sur la politique linguistique), qui remplace celle de 1983. Le nouveau document précise certains aspects de la loi précédente: si le texte de 1983 affirmait que le catalan était la langue propre de la Catalogne, celui de 1998 stipule qu'il l'identifie comme peuple.

En 2006, la version modifiée de l'Estatut d'Autonomia a été approuvée (par le Parlement catalan en 2005, puis, l'année suivante, par les Cortes Generales espagnoles, et par référendum). C'est ainsi que le Nou Estatut d'Autonomia est en vigueur depuis le mois d'août 2006; par la suite, une centaine de députés du Partido Popular (parti nationaliste espagnol) ont remis en cause la constitutionnalité de 187 de ses articles. Après trois années de débats, le tribunal constitutionnel espagnol a émis un jugement en juin 2010, qui déclara un article inconstitutionnel, treize partiellement inconstitutionnels et en clarifia 27 autres. La Catalogne est descendue dans la rue pour manifester son vif mécontentement contre la décision, qui affaiblit considérablement plusieurs dispositions du Nou Estatut d'Autonomia. Cela a pour conséquence que le catalan se voit retirer son statut de langue privilégiée de l'Administration et des communications publiques.

\subsection{L'aspect terminologique}

L'activité terminologique catalane n'est pas récente, comme l'observe Cabré ${ }^{8}$ :

On peut dire, en général, qu'en Catalogne, le développement de la terminologie est attaché aux événements historico-politiques et à la situation sociolinguistique. Sans tous ces éléments, on ne peut pas expliquer la situation actuelle. (Cabré 1989: 544)

Selon l'auteure, trois variantes déterminent le réveil terminologique catalan au début du $\mathrm{xx}^{\mathrm{e}}$ siècle: la société civile active et d'un haut niveau culturel, le nationalisme et l'industrialisation au $\mathrm{XIX}^{\mathrm{e}}$ siècle. Cette dernière permet de mettre sur pied des projets de développement de la science et de la technique par des initiatives aussi bien institutionnelles que civiles. La création en 1907 de l'Institut d'Estudis Catalans (IEC) facilite la coordination de travaux pour satisfaire les besoins terminologiques de la société. À différence d'autres académies, l'IEC n'est pas seulement chargé de normalisation, c'est aussi un organisme officiel de recherche scientifique dont l'unique idiome de travail est le catalan. 
Important philologue catalan, Pompeu Fabra dirigeait l'équipe qui a établi l'orthographe normalisée (1913), la grammaire normative (1918) et le dictionnaire de la langue (1932). Selon Cabré:

P. Fabra, qui était un ingénieur préoccupé par des technicismes et par la langue scientifique et désireux que la langue catalane soit considérée comme une véritable langue de culture au même titre que les autres langues de l'Europe, a introduit dans le dictionnaire de langue générale une quantité considérable de termes de spécialités, qui nous sont d'une grande utilité aujourd'hui. (Cabré 1989: 545)

Les activités de l'IEC ont continué clandestinement pendant l'interdiction officielle de l'idiome catalan: avec l'organisation de cours universitaires, la publication de travaux scientifiques d'intérêt terminologique et la poursuite de la codification et de l'enseignement de la langue.

En 1975, la dernière année de la dictature de Franco, un groupe nombreux de chercheurs et techniciens catalans, tous représentants de diverses sociétés, organismes institutionnels et ordres professionnels, constituèrent la Comissió Coodinadora Lexicogràfica de Ciències (commission de coordination lexicographique des sciences), dans le but d'harmoniser les néologismes techniques et scientifiques diffusés (Cabré 1989: 547).

En mai 1985 fut créée l'organisation à vocation linguistique TERMCAT (Centre de Terminologia Catalana), sur le modèle de l'Office de la langue française; M. Teresa Cabré en devient alors la première directrice. En 1986, la visite au Québec de cette dernière et d'un représentant de la Generalitat «a eu des répercussions sur l'organisation de la normalisation de la langue et la terminologie catalanes» (Cabré 2001: 133-134). Selon la même auteure, « [l]a rigueur et le prestige des modèles de politique terminologique du Québec ont inspiré et affermi les lignes directrices du modèle catalan» (Cabré 2001).

\section{Continuité des échanges}

Les sections précédentes ont montré que, si le Québec a, historiquement, pris l'initiative, la Catalogne s'est rapidement organisée elle aussi pour faire face à ses nouvelles responsabilités quant à la langue. Depuis, le Québec et la Catalogne poursuivent inlassablement leur travail terminologique et d'aménagement linguistique. Loin de demeurer unidirectionnels, les apports deviennent désormais mutuels, comme nous l'avons suggéré précédemment. Nous donnerons, dans la première partie de la présente section, divers exemples des formes que prennent maintenant les échanges québécocatalans. Ces derniers touchent les institutions, la recherche et l'enseignement.

Déjà, en 1997, la présidente du Conseil de la langue française, Nadia BrédimaAssimopoulos (1997), soulignait la valeur de l'apport catalan aux analyses québécoises:

Nous estimons que nous avons beaucoup à apprendre des communautés linguistiques qui vivent des situations similaires à la nôtre, comme c'est le cas des Catalans, et que des études de type comparatif nous aident à approfondir la réflexion sur notre propre condition.

Depuis plus de dix ans, le Conseil de la langue française entretient des relations suivies avec les principaux acteurs de la politique linguistique catalane. L'adoption en 1983 de la Loi sur la normalisation linguistique de la Catalogne a spécialement attiré notre 
attention. Cette loi nous a paru présenter de nombreuses similarités avec notre propre Charte de la langue française. Dans les deux cas, il s'agit en effet d'un projet complet d'aménagement linguistique qui vise à assurer à ces deux langues des conditions normales d'utilisation dans la vie publique. Tout naturellement, des liens se sont établis avec nos collègues, des visites ont eu lieu de part et d'autre et nous avons échangé une importante documentation sur la sociolinguistique et l'aménagement linguistique. Des experts catalans ont collaboré à certaines de nos publications et nous ont aidés à approfondir nos réflexions sur des thèmes comme la crise des langues ou les aspects politiques et juridiques de l'aménagement linguistique.

[...] La coopération entre le Québec et la Catalogne est très importante pour nous, car l'expérience catalane d'aménagement linguistique, est, sous plusieurs aspects, celle qui se rapproche le plus de notre propre expérience. (Brédima-Assimopoulos 1997)

Du reste, quelques années plus tard, un constat similaire sera émis du côté catalan par l'ex-dirigeant de la Direcció General de Política Lingüística de la Catalogne, M. Reniu i Tresserras, qui affirme que

[s]'est établi et continue à se développer un flux permanent de contacts, d'influences mutuelles, d'accords et de collaboration ouverte et cordiale entre les personnes et les organismes responsables de la politique linguistique de la Catalogne et du Québec [...]. (Reniu i Tresserras 2002: 186)

À la même époque, Cabré (2001) conclut que les deux communautés partagent une approche sociolinguistique, ainsi que l'organisation et le fondement du modèle terminologique. Selon elle, l'influence mutuelle est ressentie à divers niveaux, c'està-dire dans la méthode, les stratégies, les modes de diffusion et l'implantation.

\subsection{Influences mutuelles}

Si le constat s'avère unanime parmi les experts et les acteurs qui auront été partie prenante des nombreux échanges, à divers niveaux, entre les deux régions, la même dynamique se reflète également chez les institutions. Par exemple, le Comité mixte de coopération Québec-Catalogne, instauré en 1996, souligne l'importance des discussions sur l'aménagement et la politique linguistiques, de même que sur les lois linguistiques. De surcroît, la forte proportion de nouveaux arrivés constitue actuellement un des phénomènes communs aux deux côtés de l'Atlantique. De fait, le défi d'intégrer linguistiquement les immigrants se pose avec beaucoup d'acuité. Devant cette tâche commune, les deux régions partagent idées et expériences. En Catalogne, les immigrants - parfois même déjà hispanophones - voient dans l'apprentissage du castillan, langue internationale, un puissant levier d'intégration financière et sociale. La Catalogne faisant office de moteur économique de l'Espagne, il est logique qu'elle accueille en nombre relativement élevé les membres de cette population transplantée. Comment faire en sorte que ces expatriés adoptent le catalan? Transposée au Québec, cette question se manifeste évidemment en ce qui a trait à l'anglais: le pouvoir d'attraction de ce dernier, essentiel à la mobilité en Amérique du Nord et ailleurs dans le monde, est indéniable.

\subsubsection{Institutions gouvernementales}

L'influence mutuelle se vérifie également au niveau des institutions. Un exemple nous en est offert par Reniu i Tresserras: 
[...] les actions de la politique linguistique catalane ont influencé la politique linguistique du Québec en ce qui concerne, entre autres, la création d'un comité interministériel pour coordonner l'action de l'Administration en matière d'usage linguistique, qui se base en effet sur le Réseau technique de normalisation linguistique de Catalogne et le Consortium de normalisation linguistique. Certaines campagnes de promotion linguistique entreprises en Catalogne ont également servi de référence pour la politique linguistique québécoise. (Reniu i Tresserras 2002: 187)

À vrai dire, on n'a qu'à consulter les rapports annuels des organismes à vocation linguistique, tant québécois et catalan, pour constater l'importance accordée à la coopération internationale bilatérale. On y recense l'échange d'expertise en matière de méthodes et de critères de terminologie, ainsi que sur l'élaboration et la diffusion de vocabulaires multilingues. Dans leurs rapports annuels ou plans d'action, tant TERMCAT que l'Office québécois de la langue française ou le Secrétariat à la politique linguistique du Québec signalent mutuellement l'autre région comme partenaire privilégié. Mentionnons au passage que l'offre de services et la capacité d'innovation de TERMCAT s'avèrent remarquables depuis quelques années, signe d'un investissement constant et important de la part de l'État catalan dans ses structures terminologiques.

Catalans et Québécois se retrouvent également au sein d'organisations linguistiques et terminologiques: outre REALITER et RITERM, comptons les ententes bilatérales entre l'Office québécois de la langue française et TERMCAT et l'Institut Universitari de Lingüística Aplicada (IULA) de l'Universitat Pompeu Fabra, dans le cadre des accords de coopération gouvernementaux Québec-Catalogne.

\subsubsection{Universités}

Du côté des universités, les premiers échanges entre scientifiques catalans et québécois remontent aux années 1990; ils se sont poursuivis depuis. Ils prennent la forme de séjours postdoctoraux, de projets de recherche partagés, de collaborations en ce qui a trait à l'enseignement et de publications communes, comme en fait foi le présent article. Mentionnons au passage l'adaptation du livre La terminologia: La teoria, els mètodes, les aplicacions paru en 1992 en Catalogne, sous la plume de M. Teresa Cabré; la version française a été réalisée par des professeurs québécois et français (Cabré 1998, traduit, adapté et mis à jour par M. C. Cormier et J. Humbley). La Catalogne et le Québec échangent donc depuis plus de trente ans leurs réflexions théoriques et actions pédagogiques. L'initiative catalane se manifeste particulièrement du côté des recherches en néologie, créneau de l'IULA. La création du réseau NEOROM en constitue un brillant exemple; consacré à la néologie, il possède des antennes dans de nombreux pays latins, dont le Québec. Du côté de la terminométrie, l'influence québécoise en Catalogne sera remarquée (notamment les travaux de Auger [1999], Martin [1998] et Quirion [2003]); en revanche, on compte désormais avec l'apport catalan (Montané et Cabré 2013).

\subsection{Influence québécoise ailleurs dans le monde}

L'influence québécoise dans le domaine terminologique a faibli. Bien qu'encore très présents, les Québécois ont sans doute cédé le pas aux Catalans, qui sont devenus à certains égards, au fil des ans, le nouveau phare. Certes, le Canada et le Québec, 
représentés internationalement par le Bureau de la traduction du gouvernement fédéral et par l'Office québécois de la langue française, de même que par de nombreux professeurs et chercheurs, demeurent des joueurs importants. Citons à ce sujet Guy Dumas:

[...] plusieurs pays et communautés minoritaires ont sollicité la collaboration du Québec pour mettre à profit son expertise en matière d'élaboration de politique et de recherche linguistiques. Des ententes ont même été conclues notamment avec la Catalogne, le Pays basque et la Chine afin de tirer profit de l'expérience québécoise. Nous savons par exemple qu'en matière de recherche et de production terminologique ainsi qu'en toponymie, ces mêmes États ont transposé et parfois même adopté des concepts et une méthodologie originellement développés par les organismes linguistiques québécois chargés de l'application de la politique linguistique. (Dumas 2002: 245)

En effet, on retrouve un peu du Québec dans plusieurs pays, pour ce qui a trait à la politique linguistique, selon Dion-Viens :

Le rayonnement de la Charte de la langue française dépasse les frontières du Québec... Depuis son adoption en 1977, plusieurs États s'en sont inspirés, à commencer par la Catalogne en 1983, suivie par l'Estonie, la Belgique, le pays de Galles (Royaume-Uni), etc. Mais saviez-vous que la loi 101 a aussi servi de modèle... à la Chine et à Porto Rico ? (Dion-Viens 2007)

\subsection{Influence catalane ailleurs en Espagne et dans le monde}

Après 20 ans d'application d'un modèle d'officialisation linguistique qui se sera inspiré profondément du Québec, la Catalogne a préparé sa propre formule, qui sert maintenant à son tour de référence pour d'autres langues et qui permet désormais une relation d'échange avec le Québec.

Comment la Catalogne en est-elle venue à devenir elle-même un centre nerveux de la terminologie? C'est la question à laquelle la présente partie tentera de répondre en examinant les changements intervenus dans la dernière décennie selon plusieurs points de vue.

Sans nul doute, les actions de M. Teresa Cabré ont été déterminantes. Selon Delisle, «[1]e Québec doit aussi à cette théoricienne de la terminologie [qu'est M. Teresa Cabré] d'avoir largement diffusé ce modèle [québécois] en Espagne, en Amérique latine et en Amérique centrale» (Delisle 2008: 189).

La Catalogne a été le berceau de progrès terminologiques importants - au point de devenir un centre de référence tant en Espagne qu’à l'étranger, spécialement en Amérique latine. Les principaux axes pour lesquels la Catalogne est reconnue sont l'enseignement et la recherche en terminologie, ainsi que la production et l'organisation terminologiques.

\subsubsection{L'enseignement de la terminologie}

L'enseignement porte sur la méthodologie d'élaboration de produits terminologiques, c'est-à-dire la terminographie, et les cours théoriques et pratiques sur la discipline.

Il importe de signaler son caractère ponctuel. Il est généralement dispensé à l'extérieur du milieu universitaire, par des organisations productrices de terminologie, comme TERMCAT ${ }^{9}$. L'offre de formation de ce type a décru ces dernières années, maintenant que les futurs professionnels de la médiation linguistique (et tout parti- 
culièrement les traducteurs) fréquentent les universités. En revanche, on assiste à une augmentation de la quantité de cours ayant pour objectif la mise à jour des professionnels du secteur en lien avec la localisation et la diffusion de nouveaux produits terminologiques. Ce type de formation ne fait généralement pas partie de ceux qui sont exportés.

En ce qui concerne la terminologie, il faut préciser qu'avant 1994, cette dernière ne figurait qu'irrégulièrement aux programmes des universités espagnoles. C'est au moment de l'adoption, en 1993, d'un décret royal inscrivant les études de traduction au niveau de baccalauréat qu'elle a commencé à y être véritablement intégrée. À partir de ce moment, elle a acquis une importance sans précédent en Catalogne (où elle était déjà bien présente, maillon vital du processus d'officialisation linguistique) et dans le reste de l'Espagne.

M. Teresa Cabré, qui, comme nous l'avons dit plus haut, a influencé dans les années 80 la description terminologique dans l'officialisation linguistique, fut chargée de concevoir, de mettre en place et de diriger l'Institut Universitari de Lingüística Aplicada au sein de l'Universitat Pompeu Fabra. En 1997, naissait IULATERM, un des groupes de recherches les plus connus de l'IULA et de la discipline en général. Ce qui suit décrit quelques-uns des apports de cette équipe catalane et de l'influence qu'elle a eue sur le terrain de l'enseignement du domaine.

Depuis ses débuts, le groupe IULATERM a dispensé des cours de terminologie à divers niveaux universitaires: baccalauréat (grado), diplôme d'études supérieures spécialisées (diploma universitario de postgrado), maîtrise et doctorat. Dans les premières années, ce dernier était offert par l'IULA et la terminologie y occupait une place centrale. À cette époque ont été formés de jeunes docteurs catalans et espagnols, mais également latino-américains, provenant d'États hispanophones qui connaissaient une activité terminologique plus ou moins importante: l'Argentine, le Chili, la Colombie, Cuba, le Mexique et le Venezuela. À cela s'ajoute le Brésil, pays lusophone.

Ces nouveaux diplômés, de retour dans leur pays d'origine, ont contribué au développement de la discipline, spécialement dans les universités. Plus récemment, l'IULA a décerné des doctorats à des chercheurs d'autres États américains (comme le Pérou et l'Équateur); l'objectif de ces derniers est d'adapter les mécanismes d'officialisation du catalan - incluant la terminologie - à des langues qui traversent à l'heure actuelle une phase de revitalisation linguistique: l'aymara, l'asháninka, le cinantek, le zapoteco ou le quechua.

L'influence de la Catalogne au-delà de ses frontières (spécialement en Espagne et en Amérique latine) est également due aux diverses formations et conférences que ses chercheurs ont successivement données, notamment des cours de niveau doctoral en Espagne et sur le continent américain. On doit souligner que la plupart de ces collaborations ont été rendues possibles grâce à l'existence du RITERM ${ }^{10}$, réseau ibéro-américain de terminologie créée à Caracas au Venezuela en 1988, avec le soutien de l'Union latine (Cabré 2003). Le RITERM, dont l'objectif est de favoriser les échanges et la coopération dans le domaine, réunit par des congrès, des cours et autres activités des représentants de pays hispanophones et lusophones.

En plus de la formation doctorale, la Catalogne contribue à l'enseignement aux étudiants catalans, espagnols et étrangers de niveau baccalauréat et cycles supérieurs, tant par son offre de maîtrise dans diverses spécialités que par les programmes du groupe IULATERM. Deux de ceux-ci ont concouru à la diffusion du modèle catalan 
à l'extérieur: l'école d'été de terminologie ${ }^{11}$ et le programme en ligne IUL@online ${ }^{12}$. La première s'est offerte bisannuellement de 1997 jusqu'en 2009, quand il est apparu évident que la formation en ligne était beaucoup plus pratique pour les étrangers. IUL@online a alors été créé. C’est un programme modulaire d'apprentissage à distance dans Internet. Chaque module peut être suivi de façon indépendante (cours d'introduction, diplôme portant sur la discipline et les besoins professionnels, ateliers); leur cumul mène à l'obtention d'une maîtrise en ligne en terminologie. Évidemment, les deux programmes reflètent la vision du domaine propre à l'IULA.

Toujours en lien avec l'enseignement supérieur, mentionnons que le groupe IULATERM a été à la tête du réseau ALETERM ${ }^{13}$. Ce dernier, qui a existé de 1999 à 2001, a réuni les universités de Buenos Aires, de Brasilia, de Málaga, de Salamanca, de la Universidad del Valle en Colombie et de la Universitat Pompeu Fabra de Barcelone pour former des spécialistes autonomes et capables de mener des recherches sur le lexique et les langues spécialisés, de participer à des projets terminologiques et de les planifier, ainsi que de gérer des services de terminologie et leurs infrastructures.

\subsubsection{La recherche en terminologie}

Si la Catalogne est aujourd'hui considérée comme un centre international de recherche terminologique, cela est dû en grande partie à M. Teresa Cabré qui a proposé en 1999 la Théorie communicative de la terminologie (TCT) (Cabré 1999). Cette dernière a reçu globalement un très bon accueil, particulièrement dans les pays d'Amérique latine. La TCT remet en question les présupposés normatifs des courants antérieurs, notamment ceux de la Théorie générale de la terminologie d'Eugen Wüster (Wüster 1979), qui ont fait partie durant des décennies du modèle dominant. Elle offre une vision linguistique des unités terminologiques plus en accord avec les besoins actuels, où, de surcroît, les langues qui traversent un processus de revitalisation trouvent là une référence pour l'établissement de la terminologie.

La TCT a été complétée par les travaux des membres du groupe IULATERM: thèses doctorales, projets de recherche, articles publiés dans des revues scientifiques et présentations dans des congrès internationaux ont continué d'approfondir des aspects mentionnés, mais non développés dans la proposition initiale. Un bon exemple vient du principe de variation terminologique (Freixa 2002): un des fondements de la TCT, il a été enrichi par une dizaine de thèses soutenues à l'IULA.

Dans son souci de diffusion, la Catalogne recherche des espaces de discussion avec des spécialistes espagnols et étrangers, qui prennent souvent la forme de manifestations scientifiques. Par exemple, le congrès international de terminologie de RITERM a eu lieu à Barcelone en 2004, organisée par IULATERM de concert avec des partenaires catalans: TERMCAT et les services linguistiques des universités catalanes, regroupés dans le réseau Xarxa Vives d'Universitats ${ }^{14}$.

Depuis 1997, les membres d'IULATERM tiennent bisannuellement le symposium international d'été de terminologie, rencontre interdisciplinaire de spécialistes (surtout espagnols et latino-américains) où sont débattues diverses questions. Ainsi, en 1997, le thème à l'honneur était «terminologie et modèle culturel»; en 1999, c'était la «circulation des connaissances spécialisées »; en 2003, le sujet "objectivité scientifique et langue» a été retenu. Ses thèmes ont été choisis de façon à respecter les caractéristiques des multiples cultures représentées à ces rencontres; les connaissances scientifiques y sont abordées de façon critique et par une diversité de prismes. 
Le dernier exemple de la manière dont la Catalogne diffuse à l'extérieur de son territoire son modèle terminologique, ce sont les travaux relatifs à néologie. En 1988, était créé l'Observatoire de néologie ${ }^{15}$, groupe qui, depuis 1994, est partie intégrante de l'IULA, et qui n'a cessé de colliger et d'analyser les nouveaux mots qui apparaissent dans la presse grand public, tant en catalan qu'en castillan. Ses premières années d'activité ont été consacrées à établir à la méthodologie et à lancer ses projets. La période suivante aura permis au groupe de recherche d'obtenir une forte visibilité avec ses travaux sur l'innovation lexicale, avec la mise sur pied de divers réseaux et rencontres scientifiques - comme CINEO 2008, premier congrès international de néologie dans les langues romanes ${ }^{16}$. De surcroît, quatre réseaux d'observation ont été créés: NEOXOC ${ }^{17}$ pour l'idiome catalan; $\mathrm{NEOROC}^{18}$ pour les variétés péninsulaires de castillan; NEOROM (Cabré 2006) pour les langues romanes - qui possède une antenne à l'Université Laval pour le français québécois - ; et Antenas Neológicas ${ }^{19}$ pour les variétés de castillan des Amériques. Ce dernier réseau, le plus ancien, a été créé à la fin de 2002 avec l'objectif de contribuer à une description plus étendue du vocabulaire de ces diverses variétés linguistiques et, plus fondamentalement, à une mise à jour lexicographique. À l'heure actuelle y participent des universités d'Argentine, de Colombie, de Cuba, du Chili, du Mexique et du Pérou, tous ces groupes ayant été formés en Catalogne ou depuis la Catalogne pour la recherche en néologie.

\subsubsection{Production et organisation terminologiques}

Cette dernière partie sur la diffusion du modèle catalan portera sur les aspects de production et d'organisation terminologique. De ce point de vue, l'impact est nettement moindre qu'en recherche et en enseignement, car les publications ont un intérêt forcément plus local, lié à la langue de travail, c'est-à-dire le catalan. Néanmoins, plusieurs de celles diffusées par TERMCAT ou par les services linguistiques des universités catalanes ont obtenu une certaine reconnaissance extérieure, tout comme certains logiciels élaborés pour automatiser les tâches professionnelles, comme Terminus, gestionnaire de terminologie récemment créé par le groupe IULATERM ${ }^{20}$ . Cet outil accélère l'ensemble du travail terminographique, depuis la constitution de corpus jusqu'à l'édition finale d'un vocabulaire; de plus, étant indépendant des langues, il suscite un intérêt ailleurs qu'en Catalogne, spécialement dans les universités hispanophones, qu'elles soient d'Espagne ou d’Amérique.

En ce qui a trait à l'organisation de la terminologie, la Catalogne est présente dans la plupart des réseaux et des associations disciplinaires. Des représentants de TERMCAT, de l'IULATERM et des services linguistiques des universités catalanes font partie de REALITER (réseau panlatin), de l'AETER (association espagnole), de l'AET (association européenne), de RITERM (réseau ibéro-américain), etc. Signalons au passage que des Québécois sont également liés à la plupart de ces réseaux.

\section{Conclusion}

Malgré les différences entre les contextes québécois et catalan, spécialement historiques et législatives, les similitudes ont permis aux Catalans de prendre modèle sur le Québec quand ils ont amorcé la revitalisation de leur langue.

Avec les adaptations nécessaires, les lois québécoises pour l’aménagement linguistique ont été certainement la source d'inspiration de celles de la Catalogne et des 
moyens pour les développer et les appliquer. Dans le domaine terminologique, cette influence est peut-être encore plus évidente que sur d'autres terrains, comme l'a reconnu M. Teresa Cabré, artisane au cœur de ce processus:

Une bonne part de mes connaissances et de mes travaux en terminologie est d'inspiration québécoise dans le sens le plus large du terme. L'expérience du Québec et la bibliographie des auteurs québécois ont été les piliers de ma formation en terminologie. Les travaux de collègues et amis comme Pierre Auger et Jean-Claude Boulanger [...] ou de membres du personnel de l'Office [québécois] de la langue française [...] ont influencé la formation des jeunes universitaires catalans. (Cabré 2001: 131)

Presque trente ans plus tard, il faut dire que le modèle québécois s'est avéré une source d'inspiration efficace pour les langagiers et linguistes catalans, non seulement parce que ce modèle a permis la mise en place de stratégies et de moyens de revitalisation linguistique du catalan, mais parce qu'il sert aussi de point de repère pour d'autres langues.

\section{NOTES}

* Les auteurs remercient Louis-Jean Rousseau, Tina Célestin et Johanne Maltais, membres actuels ou retraités de l'Office québécois de la langue française, ainsi que Jean Delisle, professeur retraité de l'Université d'Ottawa, pour leur collaboration.

1. Gouvernement du Québec (1983): Décret concernant l'approbation d'une entente entre le Québec et la Catalogne. Décret no 915-83. Consulté le 17 octobre 2011, <http://www.mrifce.gouv. qc.ca/document/spdi/fonddoc/FDOC_dec_2049_Decret_0915-83.pdf >.

2. Ministère Des Relations Internationales du Québec (2011a): Catalogne. Consulté le 17 octobre 2011, <http://www.mri.gouv.qc.ca/fr/relations_quebec/europe/Espagne/catalogne/ relations.asp $>$.

3. Ministère De Relations Internationales du Québec (2011): Entente de coopération entre le gouvernement du Québec et le gouvernement autonome de la Catalogne. Consulté le 17 octobre 2011, $<$ http://www.mri.gouv.qc.ca/fr/informer/ententes/pdf/1996-04.pdf >.

4. Il est de même pour le galicien et le basque sur les territoires de la Galice et du Pays basque.

5. À la question de Font: «Est-ce que le modèle d'aménagement linguistique du catalan en Catalogne est une copie du modèle du Québec?», Tina Célestin, de l'Office québécois de la langue française, répond que «[...] le modèle linguistique du catalan en Catalogne s'est inspiré du modèle du Québec sans toutefois être une copie du modèle du Québec; en effet, il a été adapté à la situation propre à la Catalogne qui doit composer avec le statut des langues officielles en Espagne, ce qui diffère du statut des langues au Canada et dans ses provinces ainsi que dans ses territoires. L'article 1 de la Charte de la langue française affirme que "[l]e français est la langue officielle du Québec." Les Québécois n'ont pas le devoir d'apprendre l'anglais alors que tous les Espagnols en Espagne ont le devoir de connaître de castillan.» (Font, 2010).

6. «En Catalogne, après une longue gestation qui réduisit les prétentions initiales du gouvernement de Convergència i Unió, directement inspirées de la loi 101 du Québec, la loi sur la normalisation linguistique (Llei de normalització lingüística, loi 7/1983, du 18 avril) fut approuvée à l’unanimité en 1983» (Reniu i Tresserras 2002: 27).

7. En catalan, on utilise le terme normalizació lingüística pour désigner ce que l'on nomme en français aménagement linguistique.

8. Pour une présentation des activités terminologiques catalanes antérieures à 1980, la lecture de Cabré (1989) est incontournable.

9. TERMCAT. Page d'accueil: Consultée le 16 octobre 2012, <http://www.termcat.cat>.

10. RITERM. Page d'accueil: Consulté le 16 octobre 2012, <http://www.riterm.net>.

11. Universitat Pompeu Fabra. Institut Universitari de Lingüística Aplicada. Grup IULATERM. Activitats: Consulté le 16 octobre 2012, <http://www.iula.upf.edu/iulaterm/tatvca.htm>.

12. Universitat Pompeu Fabra. Institut Universitari de Lingüistica Aplicada. IUL@online: Consulté le 16 octobre 2012, <http://www.iula.upf.edu/iulonlca.htm>. 
13. Union latine. ALETERM. Red de formación en terminología: Consulté le 16 octobre 2012, <http:// www.terminometro.info/ancien/b42/es/aleterm_es.htm>.

14. Xarxa Vives d'universitats. Page d'accueil: Consulté le 16 octobre 2012, <http://www.vives.org/>.

15. Universitat Pompeu Fabra. Institut Universitari de Lingüística Aplicada. Observatori de Neologia: Consulté le 16 octobre 2012, <http://www.iula.upf.edu/obneo/obpresca.htm>.

16. CINEO 2008. Page d'accueil: Consulté le 16 octobre 2012, <http://www.iula.upf.edu/agenda/ cineo_08/index.htm>.

17. Universitat Pompeu Fabra. Institut Universitari de Lingüística Aplicada. NEOXOC: Consulté le 16 octobre 2012, <http://www.iula.upf.edu/rec/neoxoc/>.

18. Universitat Pompeu Fabra. Institut Universitari de Lingüística Aplicada. NEOROC: Consulté le 16 octobre 2012 , <http://www.iula.upf.edu/rec/neoroc/>.

19. Universitat Pompeu Fabra. Institut Universitari de Lingüística Aplicada. Antenas Neológicas: Consulté le 16 octobre 2012, <http://www.iula.upf.edu/rec/antenas/>.

20. Universitat Pompeu Fabra. Institut Universitari de Lingüística Aplicada. Recursos IULA: Consulté le 16 octobre 2012 , <http://www.iula.upf.edu/recurs02ca.htm>.

\section{REFERENCES}

Auger, Pierre (1999): L'implantation des officialismes halieutiques au Québec: essai de terminométrie. Montréal: Office de la langue française.

Bastardas Boada, Albert (1987): L’aménagement linguistique en Catalogne au $\mathrm{xx}^{\mathrm{e}}$ siècle. In: Jacques Maurais, dir. Politique et aménagement linguistique. Québec/Paris: Conseil de la langue française/Le Robert, 121-158.

Bouchard, Pierre et Gaumont, Jean-Claude (1989): Aménagement linguistique en Catalogne (Normalització lingüística a Catalunya). Mission effectuée du 13 au 17 novembre 1989. Document non publié. s. l.: Office de la langue française.

Brédima-Assimopoulos, Nadia (1997): Politique linguistique et création culturelle. Conseil supérieur de la langue française. Consulté le 29 novembre 2011, <http://www.cslf.gouv. qc.ca/le-conseil/discours-et-allocutions/archives/detail/article/politique-linguistique-etcreation-culturelle/page/6/retour/223/>.

CABré, M. Teresa (1989): La terminologie catalane: bilan des activités. Meta. 34(3):544-551.

CABré, M. Teresa (1992): La terminologia: La teoria, els mètodes, les aplicacions. Barcelona: Editorial Empúries.

CABré, M. Teresa (1998) : La terminologie - théorie, méthode et applications. Traduit, adapté et mis à jour par Monique C. Cormier et John Humbley. Paris/Ottawa: Armand Colin/ Presses de l'Université d'Ottawa.

CABrÉ, M. Teresa (1999): La terminología: Representación y comunicación. Una teoría de base comunicativa y otros artículos. Sèrie Monografies, 3. Barcelone: Institut Universitari de Lingüística Aplicada, Universitat Pompeu Fabra.

CAbré, M. Teresa (2001) : L'influence de l'Office de la langue française et du Québec sur la terminologie catalane. Interventions sociolinguistiques et pratiques langagières. Terminogramme. 101-102:131-148.

CABrÉ, M. Teresa (2003) : El papel de RITERM en la terminología iberoamericana. In: Margarita Correia, ed. Terminologia e Indústrias da Língua. Actas do VII Simpósio Ibero-Americano de Terminologia RITerm. Lisbonne: ILTEC (Unión Latina, Fundaçao Calouste Gulbenkian), 69-86.

CABrÉ, M. Teresa (2006) : NEOROM, réseau d'observatoires de la néologie des langues romanes. Neologica (1):115-118.

Corbeil, Jean-Claude (1987): Vers un aménagement linguistique comparé. In: Jacques MAURAIS, Politique et aménagement linguistique, Québec/Paris, Conseil de la langue française/ Le Robert, 555-566.

Corbeil, Jean-Claude (1990) : Relation entre sentiment national et langue. In: Gaëtan Tremblay et Manuel PArÈs I MaÏCAs, dir. Autonomie et mondialisation: le Québec et la Catalogne à 
l'heure du libre-échange et de la Communauté européenne. Québec: Presses de l'Université du Québec, 321-328.

Delisle, Jean (2008): La terminologie au Canada: histoire d'une profession. Brossard: Linguatech. Dion-Viens, Daphné (26 août 2007): La loi 101 aux quatre coins du globe. Le Soleil. 12.

Dumas, Guy (2002): L'influence de l'aménagement linguistique québécois outre-frontières. Revue d'aménagement linguistique - hors série. L'aménagement linguistique au Québec: 25 ans d'application de la Charte de la langue française, 241-245.

Font, Marc (2010): Planificació lingüística i normalització de la llengua. Dos casos d'estudi: el Quebec i Catalunya. Barcelona: Facultat de Traducció i Interpretació, Universitat Pompeu Fabra.

FreIXA, Judit (2002): La variació terminològica: anàlisi de la variació denominativa en textos de diferent grau d'especialització de l'àrea de medi ambient. (Sèrie Tesis, 3). Thèse de doctorat publiée sur CD-ROM. Barcelone: Institut Universitari de Lingüística Aplicada, Universitat Pompeu Fabra. Version en ligne: consultée le 15 décembre 2013, <http://diposit.ub.edu/ dspace/handle/2445/41609>.

LECLERC, Jacques (2010) : La politique linguistique et la Charte de la langue française. In: Jacques LeCLERC. L'aménagement linguistique dans le monde. Consulté le 16 octobre 2012, <http:// www.tlfq.ulaval.ca/AXL/amnord/quebecpollng.htm>.

Martin, André (1998): Les mots et leurs doubles: étude d'implantation de la terminologie officialisée dans le domaine de l'éducation au Québec. coll. Langues et sociétés, 36. Montréal: Office québécois de la langue française.

Montané, Amor et CABré, Teresa (2013): Bases pour la construction d'une méthodologie de suivi de l'implantation terminologique. In: Jean Quirion, Loïc Depecker et Louis-Jean Rousseau, dir. Dans tous les sens du terme. Ottawa: Presses de l'Université d'Ottawa.

QUIRION, Jean (2003): La mesure de l'implantation terminologique: proposition d'un protocole. Étude terminométrique du domaine des transports au Québec, coll. Langues et sociétés, 40. Montréal: Office québécois de la langue française.

Reniu I Tresserras, Miquel (2002): Le Québec et la Catalogne. Revue d’aménagement linguistique - hors série. L'aménagement linguistique au Québec: 25 ans d'application de la Charte de la langue française, 185-187.

Streicher-Arseneault, Valérie (2010): Las políticas lingüísticas en Quebec y Cataluña: un reflejo de las dinámicas sociales. Mémoire de maîtrise non publié. Montréal: Université de Montréal. Consulté le 16 octobre 2012, <http://papyrus.bib.umontreal.ca/jspui/bitstream/ 1866/3783/4/Streicher-Arseneault_Valerie_2009_memoire.pdf >.

Streicher-Arseneault, Valérie (2011): La planificación lingüística en Québec y en Catalunya. Tinkuy.15. Consulté le 16 octobre 2012, <http://www.littlm.umontreal.ca/recherche/documents/Tinkuy15.pdf>.

Vila I Moreno, F. Xavier et Judit Freixa (1990): Language Planning in Spain. New Language Planning Newsletter. 5(2):1-6.

Wüster, Eugen (1979): Einführung in die Allgemeine Terminologielehre und Terminologische Lexikographie. Vienne: Springer. 